\title{
Case Report: Type III Monteggia Equivalent Frac- ture Successfully Treated by Open Reduction and Internal Fixation: A Report of Two Cases
}

\author{
Morteza Nakhaei Amroodi' ${ }^{1}$, Mohammad Reza Bahaeddini' ${ }^{1}$, Shayan Amiri² (D, Mansour Karimi ${ }^{1}$ (D), Pouria Tabrizian ${ }^{1 *}$ (iD)
}

1. Bone and Joint Reconstruction Research Center, Shafa Yahyaian Hospital, Iran University of Medical Sciences, Tehran, Iran.

2. Department of Orthopaedics Surgery, Rasul-e Akram Hospital, Iran University of Medical Sciences, Tehran, Iran.

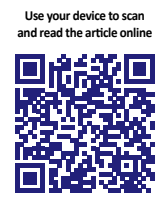

ditation Nakhaei Amroodi M, Bahaeddini MR, Amiri Sh, Karimi M, Tabrizian P. Type III Monteggia Equivalent Fracture Successfully Treated by Open Reduction and Internal Fixation: A Report of Two Cases. Journal of Research in Orthopedic Science. 2020; 7(4):175-178. http://dx.doi.org/10.32598/JROSJ.7.4.526.1

http://dx.doi.org/10.32598/JROSJ.7.4.526.1

Keywords:

Monteggia equivalent

fracture, Open reduction

and internal fixation,

Classification

\begin{abstract}
A B S T R A C T
Type III Monteggia equivalent fracture is a rare injury, and its optimal diagnosis and management are still unclear. Reporting two cases of type III Monteggia equivalent fracture in two boys who were surgically treated the day after the presentation. This study presents two cases of type III Monteggia equivalent fracture in two boys who were surgically treated the day after the presentation. These observations suggest that the timely surgical treatment of type III Monteggia equivalent fracture results in acceptable outcomes. However, the lack of a universal classification system limits the generalization of the results
\end{abstract}

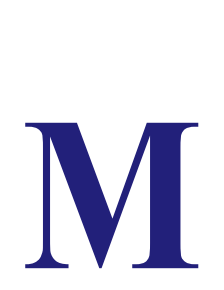

\section{Introduction}

onteggia fracture is defined as a fracture of the ulnar shaft concomitant with the dislocation of the radial head [1]. These fractures generally result from a direct blow to the forearm, when the elbow and forearm are in extended and hyperpronat- ed positions, respectively. Monteggia fractures account for $2 \%$ to $5 \%$ of all proximal forearm fractures [2].

Bado described four types of Monteggia fracture (I-IV) and a group of injuries with similar characteristics termed as Monteggia equivalents [3]. The majority of these equivalents include the variants of type I Monteggia fracture [4]. Type III Monteggia equivalent fracture occurs rarely and is described according to Bado classification,

\footnotetext{
* Corresponding Author:

Pouria Tabrizian, PhD.

Address: Bone and Joint Reconstruction Research Center, Shafa Yahyaian Hospital, Iran University of Medical Sciences, Tehran, Iran.

Phone: +98 (912) 6212291

E-mail: tabrizian.pouria@gmail.com
} 
as an ulnar fracture associated with a displaced fracture of the lateral humeral condyle [4].

In emergency departments, there is limited familiarity with these injuries, because of the low frequency of Monteggia fracture variants, particularly type III Monteggia equivalent. Therefore, more awareness is required to ensure that Monteggia equivalent fractures are accurately diagnosed and adequately managed [5].

In this study, we report two cases of type III Monteggia equivalent fractures and describe their diagnosis, management, and outcomes to raise awareness about this rare presentation.

\section{Case Presentation}

Case 1

The first case was an 8-year-old boy who was referred to the emergency department with elbow pain, the day after falling from a tree on his outstretched left hand. He had no medical history. At clinical examination, the left elbow was swollen and painful and had a limited range of motion. Besides, the plain radiographs revealed an ulnar fracture and the displaced fracture of the lateral humeral condyle, suggesting the diagnosis of type III Monteggia equivalent fracture (Figure 1) The day after the presentation, the patient underwent open reduction and internal fixation surgery.

Under general anesthesia, a Kocher approach was used to expose the elbow joint. Also, the fractures were reduced with $\mathrm{C}$-arm guidance. Then, the fractures were fixed with three pins in the lateral humeral condyle and two pins in the proximal ulna. The accuracy of fixation was checked under C-arm. After the surgery, the elbow was immobilized in an above-elbow plaster cast at 90 degrees of flexion. Also, the callus formation at the fracture sites was monitored every two weeks (Figure 1). After one month, the cast was removed and the pins were extracted. Afterward, the range of motion exercises was started. Six months follow-up of the patient was event-free. At the last follow-up, the patient regained his full range of motion with no pain.
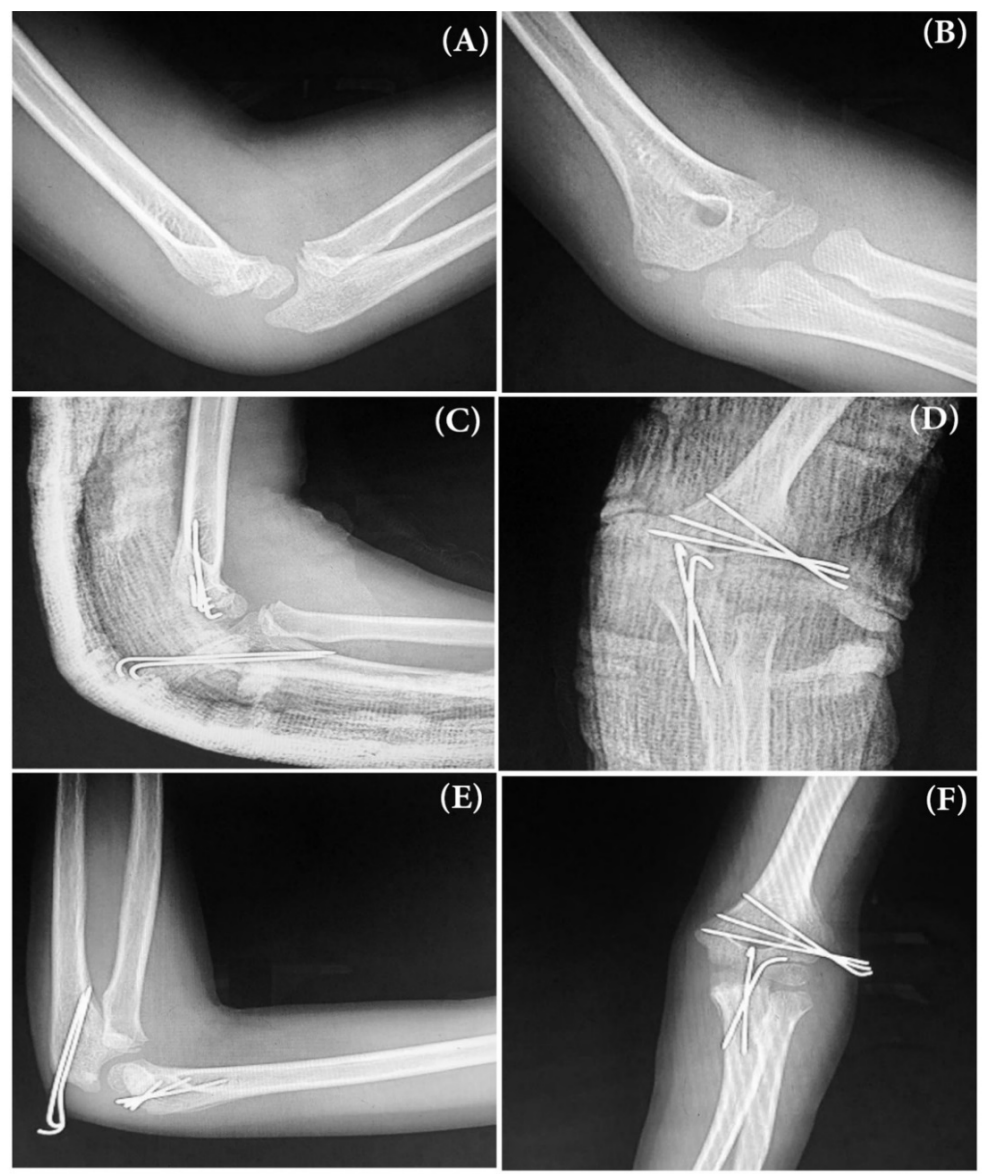

Figure 1. Anteroposterior and Lateral Radiographs of an 8-Year-Old Case of Type III Monteggia Equivalent Fracture A and B: Before the surgery; C and D: Immediately after the surgery; E and F: One month after the surgery. 


\section{Case 2}

The second case was a 5-year-old boy who was presented with the pain of the left elbow after falling from a chair. This patient had no medical history. Clinical symptoms included pain and the swelling of the left elbow with a limited range of movements. Also, the plain radiographs revealed a fracture of ulna combined with the displaced fracture of the lateral humeral condyle, which was consistent with the diagnosis of type III Monteggia equivalent fracture associated with capitulum fracture. The same surgical approach and postoperative protocol were applied for this patient, and the capitulum fracture was treated by pinning (Figure 2). Six months after the surgery, the fractures were well-united, and the elbow function was full, with no pain.

\section{Discussion}

Type III Monteggia equivalent fractures are rare injuries. Early diagnosis and treatment are essential to avoid longterm disability. In this study, we reported two cases of these fractures that were detected within one day of the injury, and surgically managed the day after the presentation. The timely diagnosis and adequate addressing of the injury components, including ulnar fracture and displaced lateral condyle fracture led to favorable outcomes in both patients. So that, both patients regained full elbow function and had no complications, in the last follow-up.

In a retrospective study, Yu et al. investigated the classification and management of Monteggia equivalent fractures in 35 children. Type III Monteggia equivalent fracture (ulnar fracture and/or olecranon fracture with humeral lateral condylar fracture) was identified in 10 cases with a mean age of 7.5 years. Also, tumbling was the main cause of injury. Furthermore, all the injuries were treated by open reduction either with internal fixation or with external fixation. All the fractures were healed after 2.5 months on average. The treatment results were excellent, good, and fair in 29,4 , and 2 cases, respectively. The authors concluded that surgery was an effective treatment for the Monteggia equivalent fractures [6].
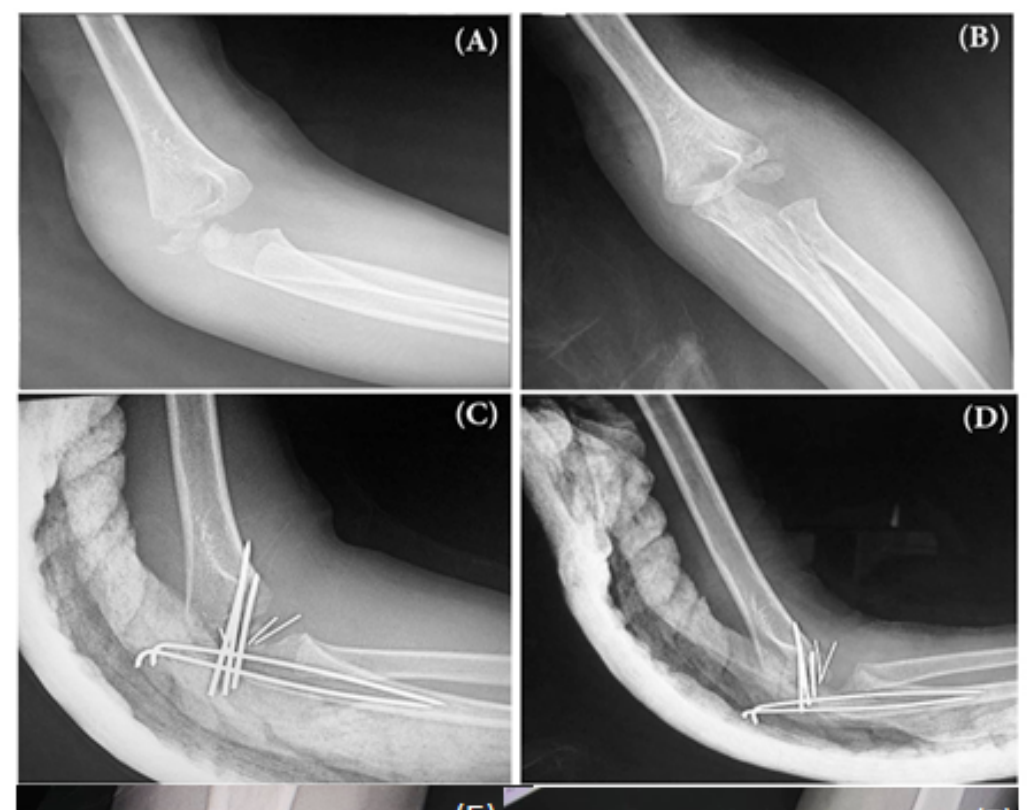

\section{)

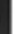

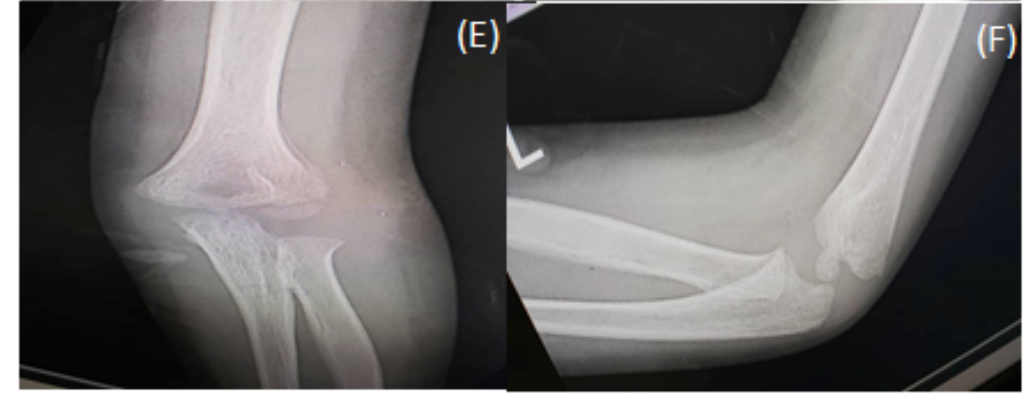

Figure 2. Radiographs of a 5-Year-Old Case of Type III Monteggia Equivalent Fracture

Orthopedic Science

A and B: Anteroposterior and lateral, before the surgery; C: Lateral, immediately after open reduction and internal fixation; D: Lateral, one month after the surgery; E and F: Anteroposterior and lateral, after pin removal (2 months after the surgery). 
Pal et al. retrospectively analyzed the outcomes of 18 patients with Monteggia equivalent fracture. Based on Bado classification, three cases of this series were classified as type III Monteggia equivalent fracture, while the Mason classification identified 10 cases with the same type of fracture. At the final follow-up, the treatment results were excellent, good, fair, and poor in 10, 4, 2, and 2 patients, respectively. The outcome of patients with type III Monteggia equivalent fracture (based on Bado classification) was excellent in two cases and good in the other one. These researchers suggested that associated fractures should be recognized accurately before the surgery to achieve optimal surgical planning, stable fixation, and early mobilization. Besides, they attributed the poor results to intra-articular injuries, coronoid fractures, and the comminution of the fractures [7].

The reports of type III Monteggia equivalent fracture are scarce. Bugeja et al. reported a 4-year-old girl with type III Monteggia lesion associated with ipsilateral humeral condyle fracture that occurred after falling from a height of two meters. The radial head dislocation was managed with closed reduction. Moreover, the ulnar fracture was fixed with percutaneous Kirschner wire. The fracture of the lateral humeral condyle was managed with open reduction and internal fixation using Kirschner wire. The patient had an excellent outcome and no complication. The authors recommended early operative treatment for these complex fractures [8]. Inconsistent with the study of Bugeja et al., the radial head was not dislocated in the present case.

Anshuman et al. reported an 8-year-old child presented with a distal one-third fracture of radius and the segmental fracture of ulna complicated with compartment syndrome. They classified this case as a type III Monteggia equivalent fracture. This classification reveals that Monteggia equivalent fractures are not clearly defined yet, and a universal classification method is required for these lesions.

\section{Conclusion}

Based on the present cases, the timely surgical treatment of type III Monteggia equivalent fracture results in acceptable outcomes. However, the lack of a universal classification of Monteggia equivalent fracture limits the generalization of the results.

\section{Ethical Considerations}

\section{Compliance with ethical guidelines}

This study ethically approved by the Ethics Committee of Iran University of Medical Sciences. Also this study was conducted based in the ethical guidelines of the Dec- laration of Helsinki, 1975. A written consent form was obtained from the participants before the study.

\section{Funding}

This research did not receive any specific grant from funding agencies in the public, commercial, or not-profit sectors.

\section{Authors' contributions}

All authors contributed in preparing all parts of the research.

\section{Conflict of interest}

The authors declared no conflict of interest.

\section{References}

[1] Monteggia GB. Istituzioni chirurgiche. Oliva; 1857. https:// books.google.com/books?id=TJrp3b6ZKYQC\&dq=Istituzioni+ chirurgiche\&source=gbs_navlinks_s

[2] Korner J, Hoffmann A, Rudig L, Müller LP, Hessmann M, Lill $\mathrm{H}$, et al. Monteggia injuries in adults: Critical analysis of injury pattern, management, and results. Unfallchirurg. 2004; 107(11):1026-40. [DOI:10.1007/s00113-004-0825-8] [PMID]

[3] Bado JL. The Monteggia lesion. Clinical Orthopaedics and Related Res. 1967; 50:71-86. [PMID]

[4] Purushothamdas S, Gayner AD. Type I Monteggia equivalent injury with posterolateral dislocation of elbow and distal epiphyseal injury-an unusual injury pattern. A case report. Eur J Orthop Surg Traumatol. 2006; 16(4):362-5. [DOI:10.1007/s00590-006-0100-5]

[5] Wich MK-HW. Monteggia and Monteggia variant fractures. In: Horwitz DS, Suk M, Swemson TK. Tips and Tricks for Problem Fractures. Berlin: Springer; 2020. [DOI:10.1007/978-3-030-38274-2_8]

[6] Yu T, Zuo Y, Wang Y, Zhou H, Wang G, Yi F, et al. Classification and treatment of Monteggia equivalent fractures in children. Zhongguo Xiu Fu Chong Jian Wai Ke Za Zhi. 2013; 27(11):130912. [PMID]

[7] Singh AP, Dhammi IK, Jain AK, Raman R, Modi P. Monteggia fracture dislocation equivalents-analysis of eighteen cases treated by open reduction and internal fixation. Chin J Traumatol. 2011; 14(4):221-6. [DOI: 10.3760/cma.j.js sn.1008-1275.2011.04.005] [PMID]

[8] Bugeja M, Avakyan A, Bianco EZ, Azzopardi T. Type III Monteggia equivalent lesion with ipsilateral fracture lateral condyle of humerus in a four-year-old child: A case report and literature review. J Orthop Case Rep. 2018; 8(5):19-21. [DOI: 10.13107/ jocr.2250-0685.1190] [PMID] [PMCID] 